\title{
Hepatocarcinoma: estado actual
}

\author{
Hepatocellular carcinoma: current situation
}

\author{
Noel Pin Vieito, Alberto Guerrero Montañés, Manuel Delgado Blanco \\ Servicio de Aparato Digestivo. Complexo Hospitalario Universitario A Coruña. SERGAS. A Coruña
}

\begin{abstract}
Resumen
El carcinoma hepatocelular $(\mathrm{CHC})$ es la neoplasia primaria hepática más frecuente. Constituye el sexto tumor más habitual en el mundo, con más de 500.000 casos diagnosticados cada año y es la tercera causa más común de muerte por cáncer.

Esta enfermedad afecta de manera casi exclusiva a pacientes con hepatopatía crónica que han desarrollado cirrosis, especialmente la relacionada con los virus de la hepatitis B y C, siendo en este grupo de sujetos el $\mathrm{CHC}$ la causa más frecuente de muerte.

La incidencia del hepatocarcinoma varía ampliamente a lo largo de la geografía mundial. Se han descrito tasas de 2 a 50 casos por 100.000 habitantes al año, en correlación a la epidemiología de sus factores de riesgo y la distribución de los diversos genotipos virales. Las zonas más prevalentes son el Sudeste Asiático y el África Subsahariana, donde la infección por el virus de la hepatitis B (VHB) es endémica. Los estudios más recientes objetivan un aumento mundial en la incidencia del $\mathrm{CHC}$, incluyendo los países occidentales, debido a la mayor diseminación del virus de la hepatitis $\mathrm{C}$ (VHC) en las décadas de los 60 y 70 además de un aumento en el consumo excesivo de alcohol.

La elevada complejidad de esta neoplasia y las patologías con las que se haya íntimamente relacionada, hacen necesario un abordaje multidisciplinar para su manejo, implicando a múltiples especialidades tanto médicas como quirúrgicas.
\end{abstract}

\section{Epidemiología}

Se han estratificado las diversas regiones del mundo en función del riesgo de desarrollo de CHC. Las áreas de elevada incidencia se caracterizan por presentar un número superior a 15 casos por 100.000 habitantes/año: África Subsahariana y regiones de Asia como Taiwan y China. Aquéllas regiones de incidencia baja están definidas por presentar un número inferior a 5 casos por cada 100.000 habitantes-año: América, Australia, Oriente próximo y la mayor parte de Europa incluyendo España. En el intervalo entre ambas se encuentran las regiones consideradas por presentar una incidencia media: Europa del Este, Haiti, Indonesia, Jamaica, Nueva Zelanda².

La incidencia del CHC es superior en los hombres. Esta diferencia es más marcada en aquellas regiones de incidencia elevada, donde se llega a alcanzar una proporción cercana a 4:1 en favor de los hombres con respecto a las mujeres ${ }^{1}$. Estas diferencias se han puesto en relación no sólo con la epidemiología de los factores de riesgo, sino también con un posible efecto trófico androgénico.

El principal factor de riesgo para el desarrollo de CHC es el VHB, asociado o no a aflatoxina. Entre el 10 y el 30\% de los CHC relacionados con VHB se desarrollan sobre hígado no cirrótico².

\begin{abstract}
Hepatocellular carcinoma ranks among the most common primary liver malignancy and the sixth most frequently diagnosed cancer worldwide. More than 500.000 cases are diagnosed each year making this pathology the third leading cause of cancer-related death in the world.

This disease affects almost exclusively to chronic liver patients who have developed cirrhosis, especially the one related to hepatitis $B$ and $C$ virus infection. Hepatocellular carcinoma is the leading cause of death in this group. The incidence of Hepatocellular carcinoma varies widely according to geographic location. Incidence rates from 2 to more than 50 cases per 100.000 inhabitants per year have been described. These differences in the distribution of $\mathrm{HC}$ are probably due to regional variations in the exposure to different risk factors and viral genotypes. The most prevalent areas include Southeastern Asia and Sub-Saharan Africa where hepatitis B viral infection is endemic.

The hepatocellular carcinoma incidence is increasing worldwide. This includes western countries due to the increased spread of hepatitis $C$ virus infection in the mid 1960s and 1970s, combined with an increase in excesive alcohol consumption in that area.

The high complexity that distiguish both this neoplasm and the pathology which has been closely related to, deserves a multidisciplinary approach with multiple medical and surgical specialties to guarantee an appropiate management.
\end{abstract}

En pacientes infectados por el VHB se ha establecido que la carga viral y la presencia de los antígenos HBe y HBs son factores de riesgo, independientes de la cirrosis, para la aparición de este tumor.

En un estudio de pacientes con VHB, naturales de Taiwan, se ha comunicado que la incidencia acumulada de $\mathrm{CHC}$ en un periodo de 11 años varía entre un 1,3\% a un 15\% en sujetos con cargas virales menores de 300 y mayores de 1 millón de copias/mL respectivamente ${ }^{4}$.Ninguno de los pacientes estaba infectado por el VHC y únicamente un 2\% de ellos tenía cirrosis. El riesgo de desarrollar el tumor se mantiene discretamente elevado $(0,06 \%)$ en portadores inactivos del VHB (sujetos HBs positivos y HBe negativos) e incluso en pacientes tratados que han perdido el antígeno HBs quienes presentarán una tasa de incidencia de 37/100.000 personas/año ${ }^{5}$.

En los países desarrollados, donde la infección por VHB no es tan prevalente, el CHC se asocia con más intensidad a la infección crónica por VHC y al consumo crónico de alcohol. En cualquier caso, se está observando una disminución de la incidencia global del $\mathrm{CHC}$ relacionado con el VHB debido a la implementación de la vacunación universal contra el virus $B$ y un aumento de los casos relacionados con el VHC y el alcohol por un mayor consumo del mismo. 
El riesgo de desarrollo de $\mathrm{CHC}$ se correlaciona con el grado de afectación hepática. Los pacientes con hepatitis crónica sin fibrosis significativa poseen un riesgo de un $1 \%$ anual que se eleva hasta un 3-7\% cuando la enfermedad progresa a una cirrosis establecida. En cualquier caso, aunque el desarrollo de $\mathrm{CHC}$ se da casi exclusivamente en pacientes con hepatopatía avanzada, hay un $10 \%$ de sujetos con $\mathrm{CHC}$ que sólo tienen fibrosis leve al diagnóstico ${ }^{6}$.

A diferencia de lo que ocurre en el VHB, donde la aparición de oncogenes específicos induce el desarrollo del tumor, en el VHC el hepatocarcinoma está fuertemente correlacionado con el grado de inflamación y necrosis hepática. El rápido recambio celular y la inflamación persistente provocada por el virus facilitaría la proliferación de hepatocitos pobremente diferenciados, el desarrollo de nódulos displásicos y finalmente el hepatocarcinoma ${ }^{7}$.

A pesar de que el mayor riesgo de $\mathrm{CHC}$ se produce en pacientes con hepatopatía crónica secundaria a VHB, VHC y hemocromatosis, cualquier enfermedad que pueda desencadenar una cirrosis hepática pone al paciente en riesgo de desarrollar un $\mathrm{CHC}$. Se ha descrito en los últimos años que aquellas condiciones que se encuentran dentro del llamado "síndrome metabólico" (obesidad, diabetes mellitus, dislipemia, resistencia a la insulina) se asocian a una mayor mortalidad relacionada con el $\mathrm{CHC}^{8}$. El tabaco también se ha asociado a un incremento de riesgo mientras que el consumo de café por el contrario parece que lo disminuye ${ }^{9,10}$. Otras sustancias sometidas a estudio como la soja, suplementos vitamínicos o medicinas alternativas como la aleta de tiburón, no han demostrado ningún efecto preventivo.

La prevención de muerte por $\mathrm{CHC}$, estará dirigida a evitar la adquisición de los diversos factores de riesgo antes mencionados. La vacunación universal contra el VHB ha demostrado ser una medida eficaz, al igual que la prevención y el tratamiento de los distintos factores de riesgo (infección por VHC, síndrome metabólico, consumo de alcohol o ingestión de aflatoxina) con campañas destinadas a mejorar los hábitos saludables de la población ${ }^{11}$.

Una vez que se produce la lesión hepática en forma de cirrosis, la eliminación del agente causal disminuye pero no elimina totalmente el riesgo de aparición de CHC.

\section{Historia natural y programas de cribado}

La historia natural del CHC es muy heterogénea. El desarrollo del hepatocarcinoma se produce habitualmente en estadios avanzados de la enfermedad hepática en aquellos pacientes infectados por el virus de la hepatitis C. Se considera que el intervalo de tiempo entre la adquisición del VHC y el desarrollo de hepatocarcinoma es de 20 a 30 años.

El virus de la hepatitis B en cambio, conduce a la aparición de esta neoplasia a través de mecanismos directos e indirectos debido a su capacidad de integración en el genoma del huésped. Esto da lugar a alteraciones del control del crecimiento celular, provocando que la aparición del CHC sea menos predecible, desarrollándose hasta en un $5 \%$ de los casos en hígados sin cirrosis ${ }^{12}$.
En algunos casos este tumor presenta un largo periodo de incubación subclínico hasta que aparece como una masa solitaria detectable en ecografía. Otras veces debuta como múltiples nódulos con tasas variables de crecimiento. La presencia de varios nódulos puede deberse a metástasis del hepatocarcinoma inicial o bien a varios tumores distintos que se desarrollan de forma sincrónica. Esta última situación es más frecuente en pacientes infectados por el $\mathrm{VHB}^{13}$.

El único método que ha demostrado eficacia en el diagnóstico precoz del CHC, es la inclusión de los sujetos de riesgo en programas de cribado. En la actualidad las guías clínicas de la American Association for the Study of Liver Diseases (AASLD) ${ }^{14}$ recomiendan la realización de screening de hepatocarcinoma en determinados grupos de riesgo a través de ecografías en intervalos de 6 meses (Figura 1). La ecografía posee una sensibilidad del 94\% para la detección de CHC, aunque disminuye a sólo 63\% para el diagnóstico de lesiones precoces. El uso de otras técnicas radiológicas o la determinación de alfafetoproteína no parecen haber demostrado incrementar su eficacia. Uno de los motivos que se esgrimen para limitar los recursos en el diagnóstico precoz de hepatocarcinomas de rápida progresión, es que su detección podría no acompañarse de una mejoría del pronóstico. Se conoce que el tamaño del tumor en el momento del diagnóstico no predice la evolución de la enfermedad con exactitud, dado que se ha descrito que la mediana del tiempo en el que un hepatocarcinoma duplica su masa puede variar entre uno y veinte meses. Además aunque muchos hepatocarcinomas mantienen su tasa de crecimiento estable, algunos muestran aumentos exponenciales o disminuciones de la misma a lo largo de su evolución, haciendo que sea muy difícil establecer el pronóstico de estos enfermos ${ }^{15}$.

Figura 1. Ecografía en modo B de un hepatocarcinoma de $2.7 \mathrm{cms}$ localizado en el segmento VIII hepático.

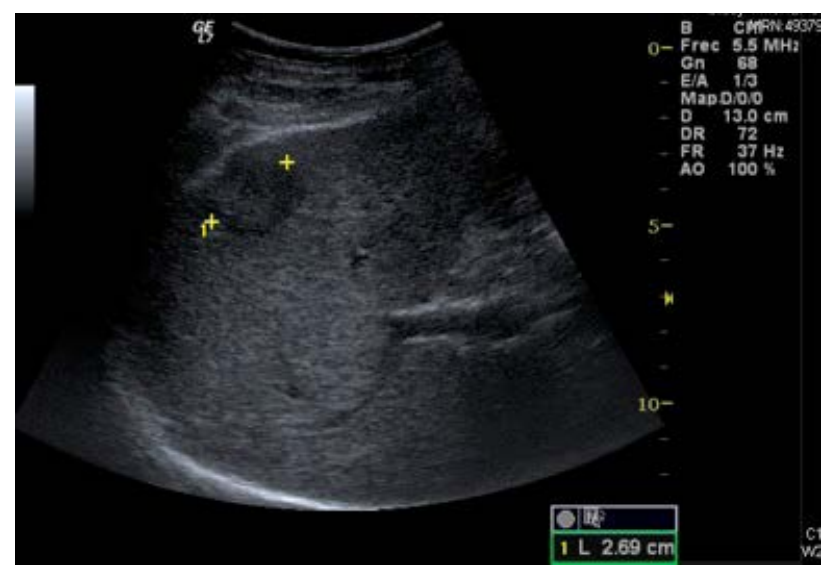

Entre los pacientes infectados por el VHB sin cirrosis, se recomienda la realización de cribado con ecografía semestral a aquellos hombres y mujeres asiáticos que tengan más de 40 y 50 años respectivamente, los africanos y personas de raza negra norteamericanos de cualquier edad, y todos los pacientes 
con antecedentes familiares de CHC desde el momento del diagnóstico. Los hombres y mujeres caucásicos con carga viral elevada (> 100,000 copias/mL) y/o transaminasas por encima de niveles normales, también deben realizar cribado por encima de los 40 y 50 años respectivamente. Además de los anteriores, cualquier paciente con cirrosis independientemente de su etiología, debe entrar en el programa de cribado ${ }^{14}$.

\section{Clínica}

La clínica del hepatocarcinoma está condicionada por la función hepática subyacente y el tamaño del tumor al diagnóstico. Éste último dependerá de la correcta inclusión previa del paciente en un programa de cribado. En estos pacientes más del $60 \%$ de CHC son diagnosticados en un estadio precoz encontrándose asintomáticos, por lo que la exploración y los hallazgos de laboratorio no serán distintos a los que encontremos en un paciente con cirrosis sin hepatocarcinoma.

El desarrollo de síntomas no suele producirse hasta que el tumor alcanza tamaños entre 4,5 y $8 \mathrm{~cm}^{16}$. Esto explica que únicamente menos del $30 \%$ de los hepatocarcinomas diagnosticados fuera de programas de cribado sean subsidiarios de tratamiento curativo ${ }^{17}$. Cuando un CHC es diagnosticado a partir de un deterioro clínico, los signos y síntomas más comunes serán hepatomegalia, molestias abdominales o el desarrollo de síndrome constitucional encontrándose todos ellos relacionados con la presencia de enfermedad avanzada.

La presencia de dolor óseo nos orienta a la presencia de metástasis a ese nivel. También debemos sospechar la aparición de un $\mathrm{CHC}$ en aquellos pacientes con una cirrosis previamente compensada que desarrollan ascitis o con menos frecuencia, sangrado digestivo por varices esofágicas y encefalopatía. Estos procesos suelen relacionarse con la presencia de invasión vascular o el desarrollo de shunts intrahepáticos ${ }^{18}$. Una complicación característica es el sangrado intraperitoneal secundario a ruptura tumoral, que se caracteriza por presentarse con gran dolor y distensión abdominal junto con un descenso importante del hematocrito.

A veces estos pacientes desarrollan síndromes paraneoplásicos, todos ellos asociados a mal pronóstico. Entre los más comunes se encuentran la aparición de hipoglucemia debida a aumento de metabolismo, hipercalcemia por secreción de proteínas relacionadas con la hormona paratiroidea o metástasis óseas, eritrocitosis por secreción de eritropoyetina o diarrea acuosa severa que se ha puesto en relación con la secreción de péptidos ${ }^{19}$.

Se pueden producir manifestaciones a nivel de la piel como dermatomiositis, pénfigo foliáceo, dermatitis seborreica o porfiria cutánea tarda entre otras, pero ninguna de ellas es específica de este tumor.

Es importante saber que la cirrosis se asocia habitualmente a la presencia de adenopatías perihepáticas inespecíficas, que no deben hacer suponer necesariamente la presencia de enfermedad avanzada en el contexto de un hepatocarcinoma.

\section{Diagnóstico}

Cualquier lesión hepática sólida, descubierta de manera incidental o como parte de un programa de cribado, en pacientes cirróticos o infectados por el VHB es altamente sugestiva de corresponder a un $\mathrm{CHC}$, y debe ser considerada de esa forma hasta que se demuestre lo contrario. La estrategia a seguir para realizar el estudio de la lesión dependerá del tamaño de la misma (Figura 2) ${ }^{20}$.

Figura 2. Manejo de un nódulo hepático detectado en el programa de cribado mediante ecografía abdominal

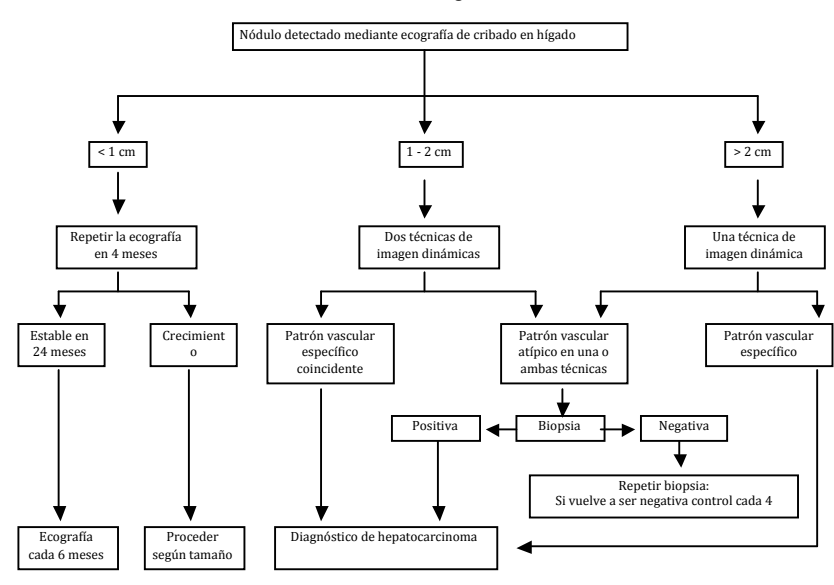

European Association for the study of the Liver 2012

En las lesiones mayores de $2 \mathrm{~cm}$, el diagnóstico de $\mathrm{CHC}$ se puede establecer por medio de un estudio de imagen con contraste bien sea una resonancia magnética (RM) 0 bien una tomografía axial computerizada (TAC) multifase (Figura 3). La realización de una biopsia no será necesaria a condición de que los hallazgos sean concordantes con el comportamiento típico de este tumor.

Figura 3. Tomografía axial computerizada que muestra una lesión hipercaptante en fase arterial, de $4.4 \mathrm{cms}$ en el segmento II hepático.

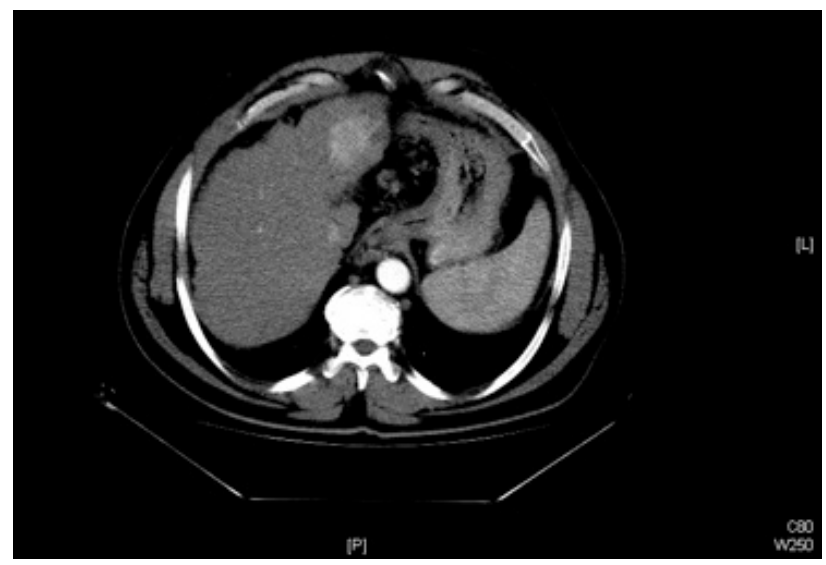

El hepatocarcinoma está irrigado solo por ramas de la arteria hepática, a diferencia del parénquima hepático que tiene un aporte mixto, compartido con las ramas de la porta. En la fase arterial de las pruebas de imagen con contraste, la sangre arterial del parénquima hepático no tumoral estará mezclada con la que 
recibe de la porta carente de contraste, por lo que la captación del mismo será más intensa en el tumor.

En la fase portal, el contraste sólo llega a las ramas portales que irrigan el parénquima normal, por eso el tumor es hipointenso con respecto al hígado colindante. La presencia de captación en la fase arterial seguido del "lavado" de contraste en la fase portal es muy específica de $\mathrm{CHC}^{21}$.

En aquellas situaciones en las que el diagnóstico es incierto, es necesario realizar una biopsia guiada. Se ha descrito un riesgo global de diseminación del tumor durante la biopsia de un 2,7\% que deberá tenerse en cuenta a la hora de decidir la indicación de la misma, en especial si se considera la posibilidad de tratamiento curativo ${ }^{22}$. Si la biopsia no es diagnóstica de $\mathrm{CHC}$, deberán repetirse los estudios de imagen cada 4 meses para descartar crecimiento de la lesión.

En los nódulos entre 1 y $2 \mathrm{~cm}$ se recomienda evidenciar el patrón de captación de contraste en dos técnicas de imagen distintas (para evitar los falsos positivos de una única imagen) excepto en centros de referencia dotados con equipos de alta definición, donde un único estudio es suficiente. Si el nódulo no muestra la captación característica, debe llevarse a cabo una biopsia de dicho nódulo con posterior análisis anatomopatológico por un experto que puede repetirse si los resultados no son definitivos. En caso de que la biopsia sea negativa se recomienda control ecográfico cada 4 meses y repetir la biopsia si existe variación en las características de la lesión.

En aquellas lesiones menores de $1 \mathrm{~cm}$, debido a su escasa probabilidad de malignidad y dificultad para su correcta caracterización, se recomienda control por medio de ecografía cada 4 meses durante un año y posteriormente cada 6 meses. Si se demuestra crecimiento de la lesión, el manejo será acorde con el tamaño adquirido.

En caso de que el paciente no presente un hígado cirrótico estos criterios de imagen no son válidos, es obligado realizar biopsia para el diagnóstico del nódulo hepático.

En cuanto al uso de marcadores serológicos para el diagnóstico, la alfa-fetoproteína presenta un rendimiento muy bajo, pudiéndose encontrar elevada en sujetos cirróticos sin $\mathrm{CHC}$ y viceversa. Su valor predictivo dependerá de la prevalencia de $\mathrm{CHC}$ en el grupo de riesgo correspondiente al paciente que evaluamos. Por ello su uso en la actualidad se ha limitado a establecer el pronóstico de $\mathrm{CHC}$ establecido ${ }^{23}$.

Recientemente se está evaluando la determinación de microRNA plasmático como ayuda al diagnóstico con resultados prometedores ${ }^{24}$. Otras técnicas no han demostrado hasta la fecha aplicabilidad clínica generalizada.

\section{Pronóstico}

El hepatocarcinoma suele aparecer en el contexto de una enfermedad hepática evolucionada. Las opciones de tratamiento y por lo tanto su pronóstico, estarán condicionados no sólo por la extensión del tumor, sino también por la funcionalidad del hígado sobre el que se desarrolla, evaluada por la escala de Child Pugh entre otras ${ }^{25}$. (Tabla1).

Tabla 1. Escala de Child Pugh

\begin{tabular}{|l|c|c|c|}
\hline \multirow{2}{*}{ Parámetro } & \multicolumn{3}{|c|}{ Puntos asignados } \\
\cline { 2 - 4 } & 1 & 2 & 3 \\
\hline Ascitis & Ausente & Leve & Moderada \\
Bilirrubina & $<2 \mathrm{mg} / \mathrm{dL}$ & $2-3 \mathrm{mg} / \mathrm{dL}$ & $>3 \mathrm{mg} / \mathrm{dL}$ \\
Albumina & $>3.5 \mathrm{~g} / \mathrm{dL}$ & $2.8-3.5$ & $\mathrm{~g} / \mathrm{dL}$ \\
\hline
\end{tabular}

Se han desarrollado múltiples herramientas para establecer el pronóstico del hepatocarcinoma. Desde un punto de vista práctiCo, las dos más utilizadas son el TNM ${ }^{26}$. (sistema de estadificación por tumor, ganglios y metástasis. Tabla 2) y el propuesto por el BCLC ${ }^{27}$ (Barcelona Clinic Liver Cancer). De forma global la mediana de supervivencia de los pacientes en el momento del diagnóstico suele ser de 6 a 20 meses $^{28}$.

El TNM ha sido revisado en el año 2010, y es el único sistema que ha sido validado en pacientes tratados tanto mediante resección hepática como por trasplante. Se ha comprobado que el porcentaje de pacientes que sobreviven a los cinco años para Ios estadíos I, II y III es de 55, 37 y $16 \%$ respectivamente. Sin embargo este sistema no tiene en cuenta la función hepática o la presencia de síntomas, que son las que determinarán la posibilidad de tratamiento y con ello el pronóstico en los pacientes cirróticos.

El sistema BCLC aporta una visión más global, incluye variables asociadas al estadio tumoral, la reserva funcional hepática y la presencia de síntomas, y establece el pronóstico de acuerdo con 4 estadios que se vinculan a la posible indicación de tratamiento.

Los pacientes que se encuentran en el estadio A están asintomáticos, tienen la función hepática conservada y presentan lesiones subsidiarias de tratamiento curativo por lo que su supervivencia esperada a los 5 años es del 50 - 75\%. Dentro de este estadio, aquellos pacientes con cirrosis hepática Child $\mathrm{A}$ y lesiones únicas menores de $2 \mathrm{~cm}$ sin invasión vascular ni diseminación a distancia, constituyen un grupo especial de buen pronóstico con una supervivencia superior al $80 \%$ a los 5 años.

Los pacientes situados en el estadio $B$ no presentan síntomas, pero tienen múltiples lesiones que por su tamaño (superior a $3 \mathrm{~cm}$ ) o por su número (mayor de 3) no son subsidiarios de tratamiento quirúrgico aunque podrían beneficiarse de quimioembolización. En este grupo la supervivencia mediana se limita a 20 meses. 
Tabla 2. American Joint Committee on Cancer (AJCC) Staging System TNM

\begin{tabular}{|c|c|c|c|}
\hline \multicolumn{4}{|c|}{ Tumor primario (T) } \\
\hline Tx & \multicolumn{3}{|c|}{ No puede evaluarse el tumor primario } \\
\hline T0 & \multicolumn{3}{|c|}{ No evidencia del tumor primario } \\
\hline $\mathrm{T} 1$ & \multicolumn{3}{|c|}{ Tumor único sin invasión vascular } \\
\hline $\mathrm{T} 2$ & \multicolumn{3}{|c|}{$\begin{array}{l}\text { Tumor único con invasión vascular } 0 \\
\text { múltiples lesiones no mayores de } 5 \mathrm{~cm}\end{array}$} \\
\hline ТЗа & \multicolumn{3}{|c|}{ Múltiples lesiones mayores de $5 \mathrm{~cm}$} \\
\hline T3b & \multicolumn{3}{|c|}{$\begin{array}{l}\text { Cualquier lesión afectando una rama } \\
\text { principal de la porta o la hepática }\end{array}$} \\
\hline T4 & \multicolumn{3}{|c|}{$\begin{array}{l}\text { Invasión directa de órganos adyacentes } \\
\text { distintos de la vesícula biliar o perforación } \\
\text { del peritoneo visceral }\end{array}$} \\
\hline \multicolumn{4}{|c|}{ Ganglios linfáticos regionales (N) } \\
\hline Nx & \multicolumn{3}{|c|}{$\begin{array}{l}\text { No puede evaluarse la existencia de } \\
\text { ganglios regionales. }\end{array}$} \\
\hline NO & \multicolumn{3}{|c|}{ No metástasis ganglionares regionales } \\
\hline N1 & \multicolumn{3}{|c|}{ Metástasis ganglionares regionales } \\
\hline \multicolumn{4}{|c|}{ Metástasis a distancia (M) } \\
\hline M0 & \multicolumn{3}{|c|}{ No metástasis a distancia } \\
\hline M1 & \multicolumn{3}{|c|}{ Metástasis a distancia } \\
\hline \multicolumn{4}{|c|}{ Grupos pronósticos } \\
\hline Estadio & $\mathrm{T}$ & N & M \\
\hline | & T1 & NO & MO \\
\hline$\|$ & T2 & NO & M0 \\
\hline IIIA & Т3а & NO & M0 \\
\hline IIIB & T3b & NO & M0 \\
\hline IIIC & $\mathrm{T} 4$ & NO & MO \\
\hline IVA & Cualquier T & N1 & MO \\
\hline IVB & Cualquier T & Cualquier N & M1 \\
\hline
\end{tabular}

Los pacientes con síntomas, invasión vascular o diseminación extrahepática presentan enfermedad avanzada. Dependiendo de su reserva funcional hepática y la severidad de sus síntomas estarán situados en los estadios C o D. Aquellos pacientes catalogados como sujetos en estadio $\mathrm{C}$ podrán ser tratados con terapia sistémica y llegan a alcanzar una supervivencia mediana de 11 meses. En cambio, en el estadio D, el único tratamiento indicado es el sintomático y solo el 10\% de los pacientes sobreviven más de un año.

Aunque no existe un consenso definitivo sobre cuál es el mejor sistema para realizar un pronóstico, la Asociación Americana Hepato-Pancreato-Biliar ha recomendado el uso del TNM para predecir la supervivencia de los pacientes subsidiarios de tratamiento quirúrgico. El BCLC se considera más preciso en el estadiaje de aquellos sujetos con enfermedad avanzada sin indicación de resección hepática o trasplante ${ }^{29}$.

A pesar de que la alfa-fetoproteína no ha demostrado utilidad en el cribado del CHC, si tiene implicaciones en el pronóstico. Sus niveles se han relacionado con el tamaño del tumor y con la tasa de crecimiento. Por ello es un marcador de mal pronóstico y un factor de riesgo de recurrencia de la enfermedad ${ }^{30}$.

Otros factores que influyen en el pronóstico de los pacientes son la histología del tumor, la presencia de una forma variante del receptor de estrógenos o la infección por el virus de la hepatitis Cy B. Marcadores serológicos e histológicos adicionales se encuentran en fase de investigación y por el momento no tienen utilidad clínica.

Sin embargo el uso de la tomografía de emisión de positrones con fluorodeoxiglucosa (PET-FDG) como complemento de los sistemas actuales de estadiaje, es un tema en investigación que podría influir en la toma de decisiones sobre el tratamiento de algunos pacientes ${ }^{31}$.

\section{Tratamiento}

Se han diseñado diversos algoritmos para intentar resumir el manejo del carcinoma hepatocelular, el propuesto por el grupo de Barcelona es uno de los más conocidos (Figura 4) ${ }^{27}$. Sin embargo, no es recomendable adoptar ningún esquema general de tratamiento de esta enfermedad debido a varios motivos. Por una parte existe una gran diversidad de opciones existentes que se hayan permanentemente sometidas a investigación. Otra razón se encuentra en la variabilidad de la eficacia de las diversas técnicas apreciada en las distintas publicaciones. Finalmente, la mayor experiencia en el uso de un tratamiento concreto por parte de los especialistas de un centro de referencia, es una razón importante para dar prioridad a esa opción en ese sitio concreto, frente a otras que podrían ser igualmente válidas en otros lugares.

Como norma general es importante valorar la extensión y resecabilidad de la lesión, la reserva funcional hepática, la presencia de infección relacionada con los virus de la hepatitis B y C, así como la situación funcional del paciente. Todos esos aspectos serán determinantes para que un equipo multidisciplinar escoja la mejor opción teniendo en cuenta la experiencia local en los diversos tratamientos.

\section{Resecabilidad de la lesión}

En la segunda conferencia consenso del 2010 entre la American Hepato-Pancreato-Biliary Association (AHPBA) y la American Joint Commission on Cancer (AJCC) sobre carcinoma hepatocelular, se ha recomendado el sistema TNM como el mejor para considerar la resecabilidad de la lesión.

Para conocer la extensión local del tumor, la prueba de elección es la tomografía axial computerizada (TAC) dinámica multifase, que permite la valoración de la lesión en las fases arterial y venosa portal. Esta prueba permite la detección de hasta un 40\% más de nódulos que la TAC convenciona ${ }^{32}$. La resonancia magnética y la arteriografía podrían tener utilidad en situaciones clínicas concretas, pero en general no aportan nada a la TAC multifase si los cortes son lo suficientemente finos.

Los lugares característicos de diseminación del hepatocarci- 
Figura 4.- Algoritmo de tratamiento de hepatocarcinoma del Barcelona Clinic Liver Cancer

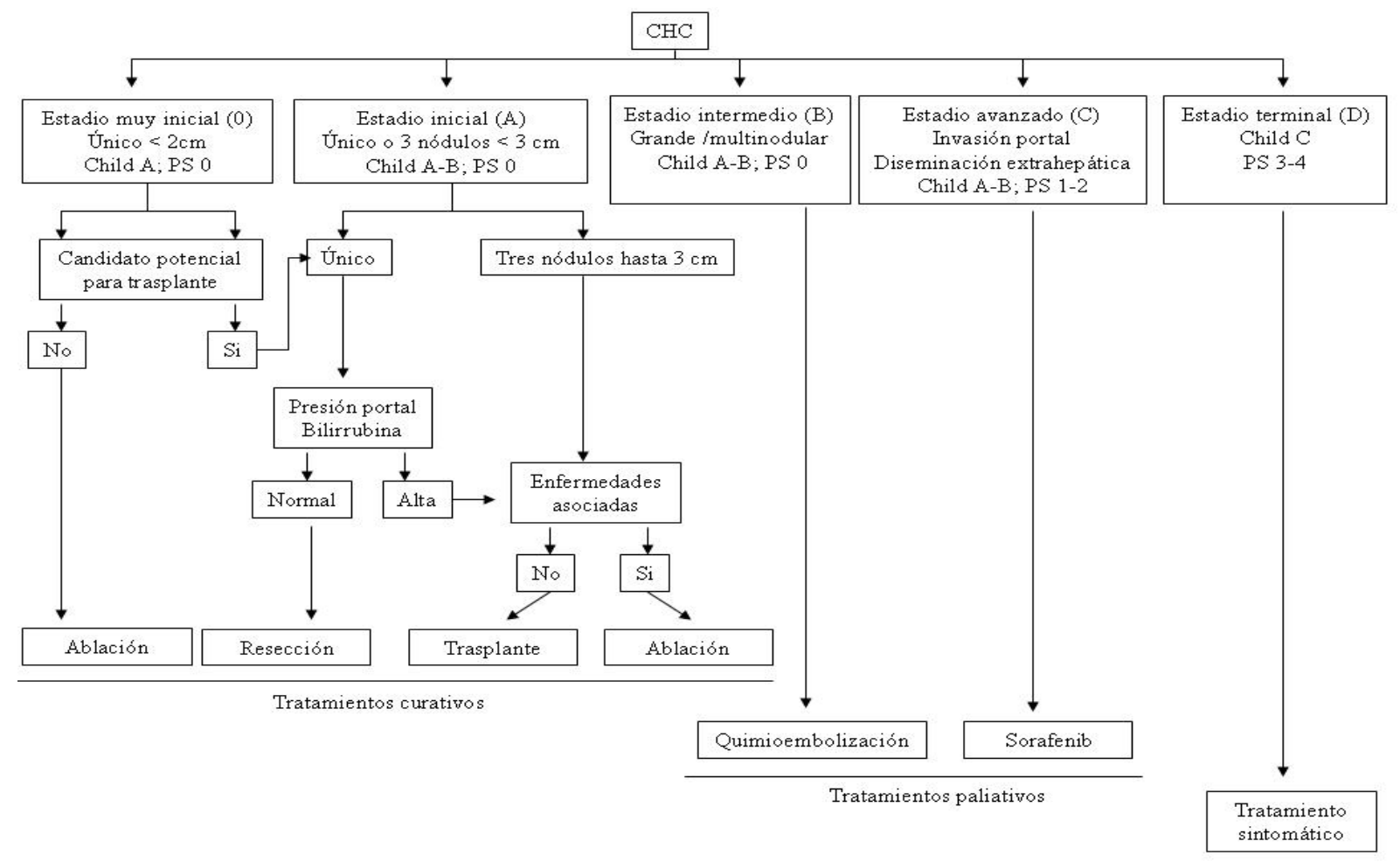

noma son pulmón, hueso, peritoneo y glándula suprarrenal. La prevalencia de enfermedad metastásica al diagnóstico es baja, por lo que no está indicado realizar un estudio rutinario dirigido a la búsqueda de lesiones extrahepáticas. Sin embargo en tumores mayores de $5 \mathrm{~cm}$ o en aquéllos que invadan estructuras vasculares la probabilidad de extensión a distancia es más elevada por lo que es necesario completar el estudio de la enfermedad mediante la realización de una TAC torácica y una gammagrafía ósea. En los pacientes valorados para la realización de trasplante también está indicado el cribado rutinario de metástasis mediante TAC torácico y TAC o RMN de abdomen y pelvis aunque la gammagrafía ósea ya no se utiliza de forma rutinaria.

Una vez realizados los estudios correspondientes, no existe consenso a la hora de definir un hepatocarcinoma como irresecable. La mayor parte de los expertos no consideran los estadíos III y IV subsidiarios de resección hepática. Estos estadíos están caracterizados por la invasión de una estructura vascular principal, un órgano distinto de la vesícula biliar, el peritoneo visceral, o la presencia de metástasis ganglionares o extrahepáticas.

El tamaño de la lesión y la multifocalidad son aspectos muy determinantes pero no excluyentes. En casos aislados, se podría considerar la resección quirúrgica en los estadios III y IV-A en algunos centros por lo que es importante insistir en la necesidad de consultar con un equipo multidisciplinar con experiencia en el manejo de la enfermedad ${ }^{33}$.

\section{Reserva funcional hepática}

La mortalidad de la cirugía del hepatocarcinoma está relacionada con la reserva funcional hepática. En los pacientes cirróticos se estima que es de un 10\%, el doble que en los pacientes con función hepática normal. La mayor parte de las muertes tras la resección hepática se deben a fallo hepático postoperatorio. La cirrosis es el predictor más importante de fallo hepático posterior a la resección y muerte.

Sólo el 5-15\% de los pacientes tendrán una función hepática adecuada que permita afrontar la resección del tumor. En los pacientes sin cirrosis, más de los 2/3 del parénquima funcional pueden ser resecados sin complicaciones, a diferencia de lo que ocurre en los pacientes cirróticos en los que la resección segura se limita a menos del 25\% del parénquima funcionante. Como norma general, los pacientes que hayan presentado complicaciones de la cirrosis como sangrado 0 ascitis o tengan hipertensión portal marcada, no poseen una reserva suficiente para afrontar una hepatectomía parcial29 .

Las escalas de Child-Pugh y MELD ${ }^{34}$ son útiles para valorar este aspecto, pero la mejor opción para determinar el volumen y la función hepática potenciales tras la resección es la realización de una volumetría hepática mediante el cálculo del aclaramiento del verde de indocianina ${ }^{35}$. Esta determinación puede tener relevancia en pacientes Child B y en aquellos Child $A$ con hepatocarcinomas que asienten en el lóbulo hepático derecho. Los pacientes Child $\mathrm{C}$ no poseen una reserva suficiente para afrontar cualquier tipo de resección.

\section{Criterios de trasplante hepático}

El trasplante hepático como tratamiento del hepatocarcinoma es una opción recomendable, ya que constituye el tratamiento del tumor y la eliminación del hígado lesionado, que está bajo riesgo de presentar nuevas lesiones. Sin embargo, el trasplante 
en lesiones no seleccionadas está asociado a recurrencias de más del $80 \%$ y bajas tasas de supervivencia con respecto a aquellos pacientes trasplantados por causas benignas con similar función hepática ${ }^{36}$.

Los criterios de Milan restringen el trasplante a aquéllos pacientes con lesiones únicas que no superen los $5 \mathrm{~cm}$ o múltiples, con un máximo de tres que no superen los $3 \mathrm{~cm}$. Además no debe existir invasión vascular, afectación ganglionar o metástasis a distancia. Si se cumplen estos supuestos el pronóstico es similar al de pacientes cirróticos trasplantados por causas benignas ${ }^{36}$. Existen algunas situaciones en las que podría considerarse el trasplante en sujetos que no cumplan estrictamente los supuestos anteriores. Estas excepciones siempre serán determinadas por grupos multidisciplinares de centros especializados y en el ámbito de la investigación. Esta actitud viene justificada porque los criterios de Milan se definieron a partir de ensayos con pacientes con hepatitis $C$, que podrían tener peor situación y pronóstico que otros cuya hepatopatía tuviera como origen el VHB o el etilismo crónico. Por ejemplo, en la Universidad de California se admiten lesiones únicas hasta 6,5 cm y múltiples (hasta tres) si ninguna es mayor de 4,5 y la suma de sus diámetros es menor de $8 \mathrm{~cm}$. En cualquier caso la inclusión en lista de un paciente de estas características nunca debe suponer un mayor tiempo de espera para otro que si cumple los criterios de Milan ${ }^{37}$.

Los hepatocarcinomas que surgen en el seno de un hígado no cirrótico, son también lesiones que no precisan cumplir de forma tan estricta los criterios de Milan.

\section{Situación funcional del enfermo}

Para estimar la calidad de vida de los enfermos de hepatocarcinoma es útil la escala ECOG ${ }^{38}$ (Tabla 3), que clasifica a los pacientes en 5 estadios dependiendo de su capacidad física. Esta escala forma parte del algoritmo utilizado por el sistema BCLC.

Tras valorar los aspectos previamente descritos, así como la seropositividad para el virus de la hepatitis B y $C$, se decidirá cual de las siguientes opciones de tratamiento es la más recomendable.

Tabla 3. Escala funcional Eastern Cooperative Oncology Group (ECOG)

\begin{tabular}{|c|c|}
\hline $\begin{array}{l}\text { Estadio } \\
\text { funcional }\end{array}$ & Definición \\
\hline 0 & Sin restricciones \\
\hline 1 & $\begin{array}{l}\text { Limitación de actividad intensa; capaz de realizar trabajo } \\
\text { ligero }\end{array}$ \\
\hline 2 & $\begin{array}{l}\text { Capaz del autocuidado pero incapaz de trabajar; se } \\
\text { levanta }>50 \% \text { del tiempo }\end{array}$ \\
\hline 3 & $\begin{array}{l}\text { Limitado para el autocuidado; Confinado a cama o sillón } \\
>50 \% \text { del tiempo }\end{array}$ \\
\hline 4 & $\begin{array}{l}\text { Completamente dependiente; Vida en cama o sillón todo } \\
\text { el tiempo. }\end{array}$ \\
\hline
\end{tabular}

\section{Resección hepática}

La hepatectomía parcial con intención curativa, es el tratamiento óptimo del hepatocarcinoma en aquellos pacientes con enfermedad localizada y buena función hepática reflejada a través de la clasificación Child Pugh o MELD.

Es necesario recordar que la resección hepática en los estadíos III y IV-A se podría valorar en centros especiales de excelencia donde alguno de estos pacientes seleccionados podría beneficiarse de este tratamiento con buenos resultados a largo plazo. Los candidatos potenciales son sujetos con un tumor localizado en una zona adecuada, con reserva funcional suficiente medida por medio de parámetros bioquímicos, y en caso de hepatectomía amplia, mediante volumetría hepática. En aquellos pacientes en los que se va a realizar una resección grande o que tienen una cirrosis severa puede intentarse embolizar la rama portal para provocar una hipertrofia del futuro remanente hepático. La supervivencia global de pacientes Child A con tumores menores de 5 cm es del $40 \%$, o del $78 \%$ en casos muy bien seleccionados ${ }^{39}$.

Dada la elevada incidencia de recidivas se ha estudiado la posibilidad de usar varios tipos de tratamiento antes o después de la cirugía. El beneficio de terapia neoadyuvante 0 adyuvante se ha estudiado en múltiples trabajos sin que por el momento se encontraran evidencias que justifiquen su uso estandarizado, aunque algunos tratamientos antivirales podrían ofrecer beneficios ${ }^{40}$.

\section{Trasplante hepático}

En los pacientes con hepatocarcinoma localizado que cumple los criterios de Milán, el trasplante es una opción apropiada. La tasa de supervivencia es superior al $75 \%$ a los cinco años. Estas cifras de supenvivencia son similares o sólo discretamente peores a las de aquellos pacientes trasplantados por causas no malignas ${ }^{41}$.

Con respecto a la "priorización" de los pacientes en lista de espera, existen varios modelos, todos ellos heterogéneos, con múltiples variables, ninguno está validado. En todos ellos el criterio fundamental es la presencia de tumor en el límite superior de los criterios de Milán (CHC T2: nódulo único de 2-5 cm 0 presencia de 203 nódulos) ${ }^{42}$.

En aproximadamente $10-20 \%$ de los casos, el tumor progresa de tal modo que el paciente deja de ser subsidiario de trasplante. Por ello en lesiones T2 que cumplen criterios UNOS de aquellas regiones donde el tiempo de espera es mayor de 6 meses por una baja disponibilidad de donantes se puede llevar a cabo un tratamiento puente con diversas opciones.

En general si existe una única lesión menor o igual a $3 \mathrm{~cm}$ se suele usar la ablación percutánea, o si la lesión es múltiple la quimioembolización ${ }^{43}$.

Además de prevenir la progresión, se ha realizado terapia alternativa al trasplante (generalmente quimioembolización) para disminuir el estadío de una lesión que previamente no cumplía 
criterios de trasplante ${ }^{44}$. Esta estrategia no está validada de forma universal, por lo que sólo debe realizarse en el seno de un ensayo clínico.

\section{Técnicas de ablación percutánea}

El tratamiento percutáneo provoca la destrucción de la lesión a través de la instilación de sustancias químicas (principalmente etanol) o mediante modificación de la temperatura intratumoral con radiofrecuencia.

Se utilizan principalmente en pacientes con grado funcional Child A o B, con lesiones en estadios iniciales donde la resección hepática y el trasplante están contraindicados. También es frecuente su empleo como tratamiento inicial en sujetos en espera de trasplante hepático para evitar la progresión tumoral durante el periodo pretrasplante.

La eficacia de este tipo de tratamiento depende del tamaño de la lesión, es máxima en las menores de $2 \mathrm{~cm}$, donde tanto la radiofrecuencia como la instilación de etanol comparten una eficacia similar que se ha equiparado a la de la resección hepática ${ }^{45}$.

En lesiones de mayor tamaño, la radiofrecuencia es más eficaz que la instilación de etanol, aunque su uso está condicionado por la localización de las mismas. El tratamiento de tumores próximos a la cúpula diafragmática 0 adyacentes a vísceras huecas se acompaña de una mayor incidencia de efectos secundarios (2-11\% de complicaciones, en especial ablación de órganos cercanos). La presencia de vasos mayores de 3 $\mathrm{mm}$ de diámetro adyacentes a la lesión puede disminuir la eficacia de esta técnica. En estas situaciones, el uso de este tratamiento mediante laparotomía abierta puede aumentar su precisión y eficacia al limitar la yatrogenia. Aunque no se ha establecido un tamaño que contraindique de forma absoluta estas técnicas, los mejores resultados se obtienen en lesiones menores de $4 \mathrm{~cm}$, siendo muy complicado destruir las de más de $6 \mathrm{~cm}^{46}$.

Se han descrito respuestas completas tras la inyección de etanol en el $80 \%$ de las lesiones menores de $3 \mathrm{~cm}$, con supervivencias superiores al $50 \%$ a los 5 años en sujetos con función hepática compensada. La eficacia en lesiones de mayor tamaño disminuye de forma progresiva en probable relación con la presencia de septos fibrosos intratumorales que impiden la adecuada difusión del etanol. Los resultados son mejores para la radiofrecuencia, aunque también disminuyen a medida que aumenta el tamaño de la lesión, $80 \%$ en tumores menores de $3 \mathrm{~cm}$ y $50-70 \%$ en lesiones de entre 3 y $5 \mathrm{~cm}^{47}$.

Al igual que en el caso de la resección quirúrgica, el principal problema de las técnicas de ablación es la elevada probabilidad de recurrencia que supera el $75 \%$ de los casos a los 5 años ${ }^{48}$.

\section{Quimioembolización}

Es el único tratamiento que ha demostrado aumentos de supervivencia en lesiones no resecables sin invasión vascular ni diseminación extrahepática.
Consiste en la inyección de un agente quimioterápico (habitualmente doxorubicina, cisplatino, epirubicina o mitomicina $\mathrm{C}$ de forma aislada o en combinación) en la rama de la arteria hepática que irriga la lesión. Este procedimiento tiene múltiples variantes en función de que se utilice o no un medio transportador para los agentes citostáticos (generalmente lipiodol), que facilitaría la retención de los mismos por el tumor, y un agente procoagulante que limitaría el aporte sanguíneo del mismo.

La existencia de múltiples formas de efectuar este tratamiento dificulta la valoración de su eficacia, aunque se estima que en más del $40 \%$ de los casos existe una respuesta mayor del $25 \% 49$. En menos del 2\% de los pacientes se consiguen remisiones completas y a pesar de ello, durante el seguimiento se producirá una restauración del flujo tumoral con posterior recidiva y reducción de la supervivencia media, inferior a los dos años tras el tratamiento. La combinación de la quimioembolización con el uso de sorafenib como tratamiento adyuvante podría enlentecer el desarrollo de recidivas ${ }^{50}$.

Los candidatos deben tener su función hepática preservada (cirrosis Child A o B no descompensada) dado que la isquemia inducida provocará, hasta en el 49 \% de los casos, daño parcial al hígado sano, lo que puede precipitar un deterioro del funcionalismo hepático. También se ha de descartar la existencia de alteraciones del flujo portal y la presencia de coagulopatía.

El efecto secundario más frecuente es el desarrollo de un síndrome postembolización (60-80\%) que se caracteriza por la presencia de fiebre, náuseas, molestias en hipocondrio derecho y elevación de la bilirrubina y transaminasas. Normalmente esta complicación se resuelve en menos de 10 días ${ }^{51}$.

Otras complicaciones menos frecuentes son el desarrollo de úlceras gastroduodenales por embolización accidental de arterias gástricas (3-5\%), disfunción renal (2\%), abscesos hepáticos (2\%) e incluso neumonías intersticiales y embolismo pulmonar y cerebral relacionados con el lipiodol.

Para minimizar los efectos secundarios se ha desarrollado el uso de esferas liberadoras de quimioterapia de forma lenta. El uso de las mismas está asociado a una menor incidencia de efectos adversos sin limitar la eficacia del tratamiento. Se han descrito respuestas cercanas al $80 \%$, superiores a la quimioembolización convencional52.

En la actualidad se investiga la posibilidad de combinar varios tratamientos locoregionales, además de otros nuevos como la radioembolización mediante esferas de Yttrium 90 que sólo se administra en el seno de ensayos clínicos.

\section{Tratamiento sistémico: Sorafenib}

El tratamiento sistémico está indicado en aquellos casos en los que están contraindicadas las terapias locoregionales debido a la presencia de invasión vascular o diseminación extrahepática.

El sorafenib es un fármaco que actúa inhibiendo múltiples vías de señalización asociadas a la carcinogénesis a través del blo- 
queo de la Raf cinasa y de diferentes tirosincinasas. Se utiliza para el tratamiento de varios tipos de neoplasias, entre ellas el hepatocarcinoma, donde actúa fundamentalmente enlenteciendo la proliferación celular y reduciendo la angiogénesis.

El sorafenib se ha evaluado en sujetos con buena reserva funcional hepática y hay pocos datos respecto al impacto en la supervivencia de sujetos con cirrosis avanzada, aunque es probable que sea bien tolerado en pacientes Child $B$ compensados, se desaconseja su uso en estadio Child C. Mediante su uso por vía oral a dosis de $400 \mathrm{mg}$ cada 12 horas, se han obtenido incrementos de la supervivencia media (10,7 meses con sorafenib frente a 7,9 con placebo) y de la mediana de tiempo de progresión (5,5 meses con sorafenib frente a 2,8 con placebo). Los efectos secundarios más frecuentes son leves y no suelen dar lugar a la retirada del medicamento. Los más típicos son diarrea, pérdida de peso y reacciones cutáneas palmo-plantares. También puede ocasionar, con menos frecuencia, toxicidad a nivel cardiaco, hepático, muscular o renal. La diátesis hemorrágica, la disfunción tiroidea, la hipertensión o el desarrollo de trombosis arterial también están descritas ${ }^{53,54}$.

La quimioterapia paliativa no se emplea de forma rutinaria. El hepatocarcinoma es un tumor considerado quimioresistente debido a la elevada frecuencia en que expresa genes asociados al desarrollo de resistencia a estos fármacos. Por otra parte la disfunción hepática condiciona la tolerancia a este tipo de tratamientos.

Aunque se han realizado múltiples ensayos con diversos agentes tanto en monoterapia como en combinación, no parece que ninguno de las pautas empleadas se asocien a aumentos de la supervivencia. Por todo ello los tratamientos con quimioterapia no están indicados.

En todos los casos en los que se emplee un tratamiento sistémico es necesario mantener el tratamiento antiviral, dado que es frecuente la reactivación del VHB.

\section{Seguimiento tras el tratamiento}

La realización de un correcto seguimiento tras llevar a cabo el tratamiento del $\mathrm{CHC}$ implica monitorizar al paciente a través de pruebas de imagen (TAC multifase o RM) y alfa-fetoproteína cada 6 a 12 meses ${ }^{55}$.

En aquellos pacientes que presentan elevación de la alfa-fetoproteína previa al tratamiento, se puede utilizar la misma como marcador de recidiva. Solo el $40 \%$ de las lesiones menores de $2 \mathrm{~cm}$ y el $30 \%$ de las que presentan un tamaño entre 2 y $5 \mathrm{~cm}$ se encuentran en esta situación.

El pronóstico tras la realización de trasplante se determinará al examinar la pieza del explante en base a la clasificación TNM. Un factor determinante es valorar la presencia de invasión microvascular.

En el caso de haber realizado tratamientos locales (quimioembolización, radiofrecuencia) es preciso determinar las medidas de la lesión pre y postratamiento. En concreto, se efectuará una TAC dinámica o bien una RM un mes después del tratamiento para evaluar la persistencia o desarrollo de un nuevo foco de captación de contraste que reflejaría la persistencia o recidiva de la enfermedad. Para poder definir de forma objetiva la situación del paciente antes y después del tratamiento, se recomienda utilizar los criterios RECIST ${ }^{56}$.

Del mismo modo, deben tenerse en cuenta las indicaciones de tratamiento antiviral y la prevención y manejo de las complicaciones de la cirrosis en aquellos sujetos con disminución de la reserva funcional hepática, en especial la profilaxis de sangrado relacionado con la presencia de hipertensión portal.

\section{Conclusión}

El hepatocarcinoma es un tumor de elevada incidencia en determinados grupos de riesgo, sobre todo en aquellos pacientes cirróticos y en los infectados por el VHB.

La vacuna de la hepatitis $B$ y la inclusión de los pacientes de alto riesgo en programas de cribado, han logrado facilitar el diagnóstico precoz de esta enfermedad haciendo posible una mejoría en el pronóstico de estos enfermos.

Es importante una adecuada valoración por grupos de expertos, formados por múltiples especialistas para decidir el mejor tratamiento a seguir a partir de las múltiples opciones existentes, entre las que destacan la resección hepática y el trasplante por sus elevadas tasas de curación.

\section{Bibliografía}

1. Jemal A, Bray F, Center MM, Ferlay J, Ward E, Forman D. Global cancer statistics, 2011. CA Cancer J Clin. 2011; 61: 69 - 90.

2. Bosch FX, Ribes J, Diaz M, Cleries R. Primary liver cancer: Worldwide incidence and trends. Gastroenterology. 2004; 127 (Suppl 1):S5-S16.

3. Davila JA, Morgan RO, Shaib Y, McGlynn KA, El-Serag HB. Hepatitis C infection and the increasing incidence of hepatocellular carcinoma: a population-based study. Gastroenterology. 2004; 127:1372 - 80.

4. Chen CJ, Yang HI, Su J, Jen CL, You SL, Lu SN et al. Risk of hepatocellular carcinoma across a biological gradient of serum hepatitis B virus DNA level. JAMA. 2006; 295:65 - 73.

5. Simonetti J, Bulkow L, McMahon BJ, Homan C, Snowball M, Negus S et al. Clearance of hepatitis $B$ surface antigen and risk of hepatocellular carcinoma in a cohort chronically infected with hepatitis B virus. Hepatology 2010; 51:153 - 7.

6. Lewis S, Roayaie S, Ward SC, Shyknevsky I, Jibara G, Taouli B. Hepatocellular carcinoma in chronic hepatitis $\mathrm{C}$ in the absence of advanced fibrosis or cirrhosis. AJR Am J Roentgenol. 2013; 200: W 610 - 6.

7. Budhu A, Wang XW. The role of cytokines in hepatocellular carcinoma. J Leukoc Biol. 2006; 80: 1197- 213.

8. Yasui K, Hashimoto E, Komorizono Y, Koike K, Arii S, Imai Yet al. Characteristics of patients with nonalcoholic steatohepatitis who develop hepatocellular carcinoma. Clin Gastroenterol Hepatol. 2011; 9: 428 - 33

9. Kuper H, Tzonou A, Kaklamani E, Hsieh CC, Lagiou P, Adami HO, et al. Tobacco smoking, alcohol consumption and their interaction in the causation of hepatocellular carcinoma. Int J Cancer. 2000; 85: 498-502.

10. Bravi F, Bosetti C, Tavani A, Bagnardi V, Gallus S, Negri E, et al. Coffee drinking and hepatocellular carcinoma risk: A meta-analysis. Hepatology. 2007; 46: 430 - 5.

11. Kew MC. Prevention of hepatocellular carcinoma. Ann Hepatol. 2010; 9: 120 - 32.

12. McMahon,B.J. Epidemiology and natural history of hepatitis B. Semin. Liver Dis 2005; 25: S3-S8.

13. Katoh H, Shibata T, Kokubu A, Ojima H, Loukopoulos P, Kanai Y et al. Genetic profile of hepatocellular carcinoma revealed by array-based comparative genomic hybridization: identification of genetic indicators to predict patients outcome. J Hepatol 2005; 43: 863 - 74.

14. Bruix J, Sherman M. AASLD practice guideline. Management of hepatocellular carcinoma: an update. Hepatology 2011; 53: 1020 - 2 
15. Cheng SJ, Freeman RB Jr, Wong. Predicting the probability of progression-free survival in patients with small hepatocellular carcinoma. Liver Transpl 2002; 8: 323 - 8.

16. Yuen MF, Cheng CC, Lauder IJ, Lam SK, Ooi CG, Lai CL. Early detection of hepatocellular carcinoma increases the chance of treatment: Hong Kong experience. Hepatology 2000; 31: $330-5$.

17. Trevisani F, De Notariis S, Rapaccini G, Farinati F, Benvegnù L, Zoli M et al. Semiannual and annual surveillance of cirrhotic patients for hepatocellular carcinoma: effects on cancer stage and patient survival (Italian Experience). Am. J. Gastroenterol 2002; 97: 734 - 44

18. Sugano S, Miyoshi K, Suzuki T, Kawafune T, Kubota M. Intrahepatic arteriovenous shunting due to hepatocellular carcinoma and cirrhosis, and its change by transcatheter arterial embolization. Am J Gastroenterol. 1994; 89:184 - 8.

19. Luo JC, Hwang SJ, Wu JC, Lai CR, Li CP, Chang FY et al. Clinical characteristics and prognosis of hepatocellular carcinoma patients with paraneoplastic syndromes. Hepatogastroenterology 2002; 49:1315-9.

20. European Association For The Study Of The Liver, European Organisation For Research And Treatment Of Cancer. EASL-EORTC clinical practice guidelines: management of hepatocellular carcinoma. J Hepatol 2012; 56: 908 - 43.

21. Yu JS, Kim KW, Kim EK, Lee JT, Yoo HS. Contrast enhancement of small hepatocellular carcinoma: usefulness of three successive early image acquisitions during multiphase dynamic MR imaging. AJR Am J Roentgenol 1999; 173: 597 - 604.

22. Silva MA, Hegab B, Hyde C, Guo B, Buckels JA, Mirza DF. Needle track seeding following biopsy of liver lesions in the diagnosis of hepatocellular cancer: a systematic review and meta-analysis. Gut. 2008; 57:1592 - 6 .

23. Ioannou GN, Perkins JD, Carithers RL Jr. Liver transplantation for hepatocellular carcinoma: impact of the MELD allocation system and predictors of survival. Gastroenterology. 2008; 134: 1342 - 51

24. Borel F, Konstantinova P, Jansen PL. Diagnostic and therapeutic potential of miRNA signatures in patients with hepatocellular carcinoma. J Hepatol. 2012; 56:1371 83.

25. Pugh RN, Murray-Lyon IM, Dawson JL, Pietroni MC, Williams R. Transection of the oesophagus for bleeding oesophageal varices. Br J Surg 1973; 60: 646 - 9.

26. Edge SB, Byrd DR, Compton CC, et al. American Joint Committee on Cancer. American Joint Committee on Cancer Staging Manual, 7th, (Eds), Springer, New York 2010. p.175.

27. Forner A; Llovet JM, Bruix J. Hepatocellular carcinoma. Lancet 2012; 379: 1245 55.

28. Bruix J, Gores GJ, Mazzaferro V.Hepatocellular carcinoma: clinical frontiers and perspectives. Gut. 2014; 63: $844-55$.

29. Vauthey JN, Dixon E, Abdalla EK, Helton WS, Pawlik TM, Taouli B et al. American Hepato-Pancreato-Biliary Association, Society of Surgical Oncology, Society for Surgery of the Alimentary Tract. Pretreatment assessment of hepatocellular carcinoma: expert consensus statement. HPB (0xford). 2010; 12:289 - 99.

30. Kumada T, Nakano S, Takeda I, Sugiyama K, Osada T, Kiriyama S et al. Patterns of recurrence after initial treatment in patients with small hepatocellular carcinoma. Hepatology, 1997; 25: 87-92.

31. Kornberg A, Küpper B, Tannapfel A, Büchler P, Krause B, Witt U et al. Patients with non-[18 F]fludeoxyglucose-avid advanced hepatocellular carcinoma on clinical staging may achieve long-term recurrence-free survival after liver transplantation. Liver Transpl. 2012; 18: 53 - 61.

32. Wald C, Russo MW, Heimbach JK, Hussain HK, Pomfret EA, Bruix J. New OPTN/ UNOS policy for liver transplant allocation: standardization of liver imaging, diagnosis, classification, and reporting of hepatocellular carcinoma. Radiology 2013; 266: $376-82$.

33. Andreou A, Vauthey JN, Cherqui D, Zimmitti G, Ribero D, Truty MJ et al. International Cooperative Study Group on Hepatocellular Carcinoma. Improved long-term survival after major resection for hepatocellular carcinoma: a multicenter analysis based on a new definition of major hepatectomy. J Gastrointest Surg. 2013; 17: 66 - 77.

34. Suman A, Barnes DS, Zein NN, Levinthal GN, Connor JT, Carey WD. Predicting outcome after cardiac surgery in patients with cirrhosis: a comparison of Child-Pugh and MELD scores. Clin Gastroenterol Hepatol. 2004; 2: 719 - 23.

35. Hemming AW, Scudamore $\mathrm{CH}$, Shackleton CR, Pudek M, Erb SR. Indocyanine green clearance as a predictor of successful hepatic resection in cirrhotic patients. Am J Surg. 1992; 163: 515-8.

36. Mazzaferro V, Regalia E, Doci R, Andreola S, Pulvirenti A, Bozzetti F et al. Liver transplantation for the treatment of small hepatocellular carcinomas in patients with cirrhosis. N Engl J Med. 1996; 334: 693-9.

37. Yao FY, Ferrell L, Bass NM, Watson JJ, Bacchetti P, Venook A et al. Liver transplantation for hepatocellular carcinoma: expansion of the tumor size limits does not adversely impact survival. Hepatology. 2001; 33:1394-403

38. Oken MM, Creech RH, Tormey DC, Horton J, Davis TE, McFadden ET et al. Toxicity and response criteria of the Eastern Cooperative Oncology Group. Am J Clin Oncol. 1982; 5: 649 - 55.

39. Shi M, Guo RP, Lin XJ, Zhang YQ, Chen MS, Zhang CQ et al. Partial hepatectomy with wide versus narrow resection margin for solitary hepatocellular carcinoma: a prospective randomized trial. Ann Surg. 2007; 245: 36 - 43.

40. Wong JS, Wong GL, Tsoi KK, Wong VW, Cheung SY, Chong CN et al. Meta-analysis: the efficacy of anti-viral therapy in prevention of recurrence after curative treatment of chronic hepatitis B-related hepatocellular carcinoma. Aliment Pharmacol Ther. 2011; 33: $1104-12$

41. Wong SN, Reddy KR, Keeffe EB, Han SH, Gaglio PJ, Perrillo RP et al. Comparison of clinical outcomes in chronic hepatitis B liver transplant candidates with and without hepatocellular carcinoma. Liver Transpl 2007; 13: 334 - 42.

42. Lladó L, Bustamante J, en nombre del grupo de trabajo de la Sociedad Española de Trasplante Hepático. IV reunión de consenso de la Sociedad Española de Trasplante Hepático 2012. Excepciones al Model for End-stage Liver Disease en la priorización para trasplante hepático. Gastroenterol Hepatol. 2014; 37: 83 - 91

43. Lin Y, Chapman WC. Towards more effective liver allocation criteria for hepatocellular carcinoma: tumor response to locoregional therapy. Ann Surg Oncol. 2011; 18: 2416 - 8.

44. Barakat O, Wood RP, Ozaki CF, Ankoma-Sey V, Galati J, Skolkin M et al. Morphological features of advanced hepatocellular carcinoma as a predictor of downstaging and liver transplantation: an intention-to-treat analysis. Liver Transpl 2010; 16: 289 - 99.

45. Weis S, Franke A, Mössner J, Jakobsen JC, Schoppmeyer K. Radiofrequency (thermal) ablation versus no intervention or other interventions for hepatocellular carcinoma. Cochrane Database Syst Rev. 2013;12:CD003046.

46. Takaki H, Yamakado K, Nakatsuka A, Yamada T, Shiraki K, Takei Y et al. Frequency of and risk factors for complications after liver radiofrequency ablation under CT fluoroscopic guidance in 1500 sessions: single-center experience. AJR Am J Roentgenol. 2013; 200: 658 - 64.

47. Yin XY, Xie XY, Lu MD, Xu HX, Xu ZF, Kuang M et al. Percutaneous thermal ablation of medium and large hepatocellular carcinoma: long-term outcome and prognostic factors. Cancer 2009; 115: 1914 - 23.

48. Shiina S, Tateishi R, Arano T, Uchino K, Enooku K, Nakagawa H et al. Radiofrequency ablation for hepatocellular carcinoma: 10-year outcome and prognostic factors. Am J Gastroenterol. 2012; 107: 569 -77.

49. Georgiades CS, Liapi E, Frangakis C, Park JU, Kim HW, Hong K et al. Prognostic accuracy of 12 liver staging systems in patients with unresectable hepatocellular carcinoma treated with transarterial chemoembolization. J Vasc Interv Radiol. 2006; 17: $1619-24$

50. Sansonno D, Lauletta G, Russi S, Conteduca V, Sansonno L, Dammacco F. Transarterial chemoembolization plus sorafenib: a sequential therapeutic scheme for HCVrelated intermediate-stage hepatocellular carcinoma: a randomized clinical trial. Oncologist. 2012; 17: 359 - 66

51. Marelli L, Stigliano R, Triantos C, Senzolo M, Cholongitas E, Davies N et al. Transarterial therapy for hepatocellular carcinoma: which technique is more effective? A systematic review of cohort and randomized studies. Cardiovasc Intervent Radiol. 2007; 30: 6 - 25.

52. Vogl TJ, Lammer J, Lencioni R, Malagari K, Watkinson A, Pilleul $F$ et al. Liver, gastrointestinal, and cardiac toxicity in intermediate hepatocellular carcinoma treated with PRECISION TACE with drug-eluting beads: results from the PRECISION V randomized trial. AJR Am J Roentgenol. 2011; 197: W562 - 70.

53. Llovet JM, Ricci S, Mazzaferro V, Hilgard P, Gane E, Blanc JF et al. Sorafenib in advanced hepatocellular carcinoma. N Engl J Med. 2008; 359: 378 - 90.

54. Reig M, Matilla A, Bustamante J, Castells L, de la Mata M, Delgado M et al. Recomendaciones de manejo de Sorafenib en pacientes con carcinoma hepatocelular. Gastroenterol Hepatol 2010; 33: 741 - 52.

55. Clavien PA, Lesurtel M, Bossuyt PM, Gores GJ, Langer B, Perrier A, OLT for HCC Consensus Group. Recommendations for liver transplantation for hepatocellular carcinoma: an international consensus conference report. Lancet Oncol. 2012; 13: e11- 22.

56. Lencioni R, LLovet JM Modified RECIST (mRECIST) assessment for hepatocellular carcinoma. Semin Liver Dis 2010: 30: 52 - 60. 